\title{
Clustering predicts memory performance in networks of spiking and non-spiking neurons
}

\author{
Weiliang Chen ${ }^{1,2 *}$, Reinoud Maex ${ }^{1}$, Rod Adams' ${ }^{1}$, Volker Steuber ${ }^{1}$, Lee Calcraft ${ }^{1}$ and Neil Davey ${ }^{1}$ \\ Science and Technology Research Institute, University of Hertfordshire, Hatfield, Hertfordshire, UK \\ 2 Computational Neuroscience Unit, Okinawa Institute of Science and Technology, Okinawa, Japan
}

Edited by:

Ad Aertsen, Albert Ludwigs University,

Germany

Reviewed by:

Bruce Graham, University of Stirling,

UK

Guenther Palm, Universit of UIm,

Germany

*Correspondence:

Weiliang Chen, Computational

Neuroscience Unit, Okinawa Institute

of Science and Technology, Seaside

House, 7542, Onna, Onna-Son,

Kunigami, Okinawa 904-0411, Japan.

e-mail:w.chen@oist.jp
The problem we address in this paper is that of finding effective and parsimonious patterns of connectivity in sparse associative memories. This problem must be addressed in real neuronal systems, so that results in artificial systems could throw light on real systems. We show that there are efficient patterns of connectivity and that these patterns are effective in models with either spiking or non-spiking neurons. This suggests that there may be some underlying general principles governing good connectivity in such networks. We also show that the clustering of the network, measured by Clustering Coefficient, has a strong negative linear correlation to the performance of associative memory. This result is important since a purely static measure of network connectivity appears to determine an important dynamic property of the network.

Keywords: perceptron, learning, associative memory, small-world network, non-random graph, connectivity

\section{INTRODUCTION}

Network models for associative memories store the information to be retrieved in the values of the synaptic weights. Weighted summation of their synaptic inputs then allows the neurons to transform any input pattern into an associated output pattern. Network models for associative memories come in two flavors. In pure feedforward models, like the one-layer perceptron, a static input pattern in the afferent fibers is in one step transformed, by weighted summation, into a static pattern of activity of the neurons in the output layer. For instance Purkinje cells in the cerebellum have been proposed to recognize in this way patterns of activity in the mossy fibers (Marr, 1969; Tyrrell and Willshaw, 1992; Steuber et al., 2007). In feedback models, which are the subject of the present paper, the memory is stored in the weights of recurrent connections between the neurons. The input pattern (one input for each neuron) initializes the neurons, after which the network is left free to run until the neuronal activities potentially converge to a stable pattern, which no longer changes over time. The main advantage of feedback networks is that they can work self-correctively: noisy input patterns can converge to the original attractor pattern. The main questions are how a desired memory can be stored by making it an attractor of the network (the learning rule determining the synaptic weights) and how many patterns can be stored and retrieved, within a given error margin, in a network with a given number of neurons and synapses.

These questions were solved analytically for the so-called Hopfield network (Hopfield, 1982), which, however, takes several simplifications that are biologically unrealistic. The Hopfield network, in its original formulation, consists of two-state (bipolar) neurons that are fully connected to each other, with each pair connected bi-directionally with identical weights. More biologically realistic adaptations of the Hopfield network, using sparse and non-symmetrical connections, have been shown to have, like the perceptron, a capacity of $2 k$ patterns, $k$ being the number of connections to each neuron (Diederich and Opper, 1987; Gardner, 1988).

In the present paper, building on our previous work (Davey and Adams, 2004; Calcraft et al., 2006, 2007, 2008; Davey et al., 2006; Chen et al., 2009), we use computer simulations to address three other biological constraints for sparsely connected networks. Firstly, how does the topology of the wiring pattern affect memory performance? Secondly, can memories be retrieved by a network of spiking neurons with connection delays? Thirdly, taking into account wiring length, which wiring pattern has the highest cost efficiency?

\section{MATERIALS AND METHODS THE NETWORK MODEL FOR ASSOCIATIVE MEMORY Network connectivity}

For each model investigated, a collection of $N$ artificial neurons is placed on a line such that the nodes are equally spaced. To avoid edge effects we consider the ends of the line to meet, so that we have a so-called periodic boundary. This gives the network a ring topology. For simplicity we define the distance between neighboring neurons to be 1 . To aid in visualization we represent the networks as a ring, but they are always actually 1-D. Once the neurons have been positioned they can be connected to one another in a variety of fashions. However, there are three common features shared among all network models. Firstly, the networks are regular, so that each neuron has $k$ incoming connections. To maintain this fan-in during rewiring, only the index of the source (afferent) neuron of a connection could be changed. Therefore although the connection strategy varies in the present study, the values of $N$ and $k$ are constant. Secondly, the networks are sparse, so that with a network of $N$ units, $k \ll N$, resembling the sparse connectivity of 
the mammalian cortex. Thirdly, the connections are directed, so if the connection from $i$ to $j$ exists, this does not imply that the reciprocal connection from $j$ to $i$ also exists.

With this configuration, two extreme cases are widely known and commonly studied. The first case is a completely local network, or lattice, whose nodes are connected to those nodes that are closest to it. An example of a local network is the cellular neural network (CNN), where units are connected locally in 2-D (Brucoli et al., 1996). Alternatively the network can be connected in a completely random manner, where the probability of any two nodes being connected is $k / N$, independently of their position. Locally connected networks have a minimum wiring length, but perform poorly as associative memories, since pattern correction is a global computation and full local connectivity does not allow easy passage of information across the whole network. In randomly connected networks, on the other hand, information can readily move through the network, and consequently pattern correction is much better and in fact cannot be improved with any other architecture. However, in real biological networks such as cerebral cortex, completely random connectivity suffers from the restriction of high wiring cost and therefore may not be the desirable choice. An optimized connectivity is one that gives a performance comparable to random networks, but with more economical wiring. The existence of such connectivity, as well as its construction strategy, has been of great interest in recent research. One family of network connectivities, the so-called small-world networks (Watts and Strogatz, 1998), has been suggested to exhibit this optimization.

Our first connection strategy is adapted from the method proposed by Watts and Strogatz (1998). The Watts-Strogatz smallworld network starts from a local network, where each unit has incoming connections from its $k$ nearest neighbors (to simplify results, networks studied in this paper have no self-connectivity). The local network is then progressively rewired by randomly reassigning a fraction $(p)$ of the afferent neurons. The network is transformed into a completely random network when $p$ reaches 1 (Figure 1). In the rest of the paper we refer to networks constructed in this way as small-world networks.

Another connection strategy investigated by us exhibits Gaussian connectivity, where each unit has $k$ incoming connections from neurons whose distances form a Gaussian distribution (see Figure 7). The connectivity of such a network is parameterized by the SD of its distance distribution, $\sigma$. We have shown in earlier work (Calcraft et al., 2007) that both small-world and Gaussian networks can perform optimally as associative memories and that tight Gaussian distributions give very parsimonious networks with efficient use of connecting fiber.

The biological context of this connectivity comes from the mammalian cortex, which is thought to have a similar connectivity between individual neurons (Hellwig, 2000).

The modularity of mammalian cortex, commonly referred to as the hypothesis of cortical columns (Mountcastle, 1997), is also considered in our investigation. Three different types of modular connectivity are studied. The first one, named the Fully Connected Modular network, contains $m$ internally fully connected subnetworks, defined as modules. Initially there is no interconnection between modules, thus each module can be treated as an isolated

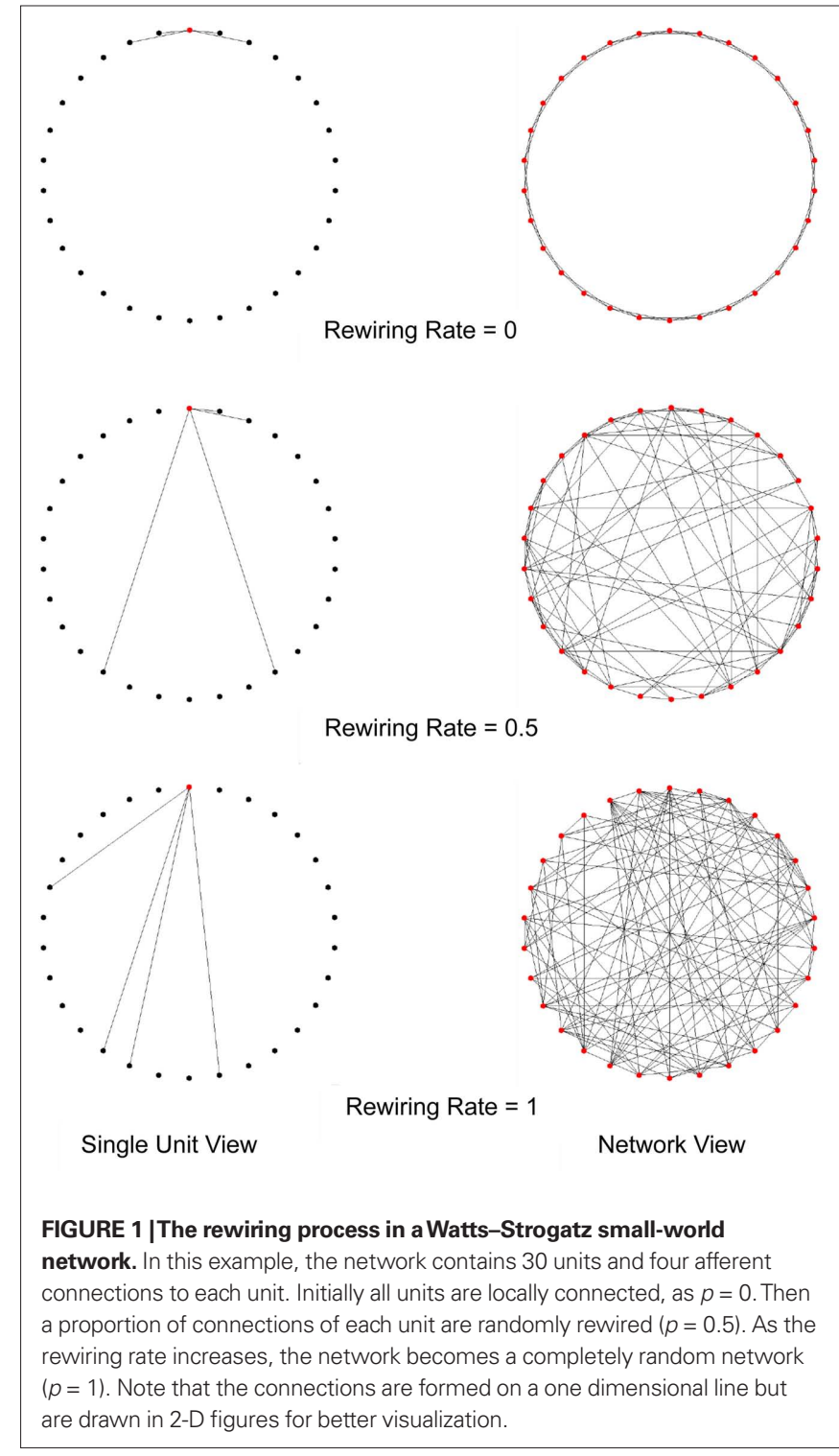

fully connected associative memory model. The subnetworks establish connections by randomly rewiring the intra-modular connections to form random connections from anywhere across the whole network. A fraction $p$ denotes the proportion of rewired connections. The network has the same type of connectivity as the Watts-Strogatz small-world network, when $p$ reaches 1 . Figure 2 gives an example of networks with this connectivity.

Another modular network we have investigated is called the Gaussian-Uniform Modular Network. In such a network, the intra-modular connections within each module have a Gaussiandistributed connectivity with a SD of $\sigma_{\text {internal }}$, which is proportional to the number of internal connections per unit, $k_{\text {internal }}$. Each unit in the network also has a number of external connections (defined by $\left.k_{\text {external }}\right)$ from units in other modules. The connectivity of the external network is uniformly random. Although $k_{\text {internal }}$ and $k_{\text {external }}$ vary in different configurations, the total number of connections per unit, $k=k_{\text {internal }}+k_{\text {internal }}$ is maintained so that the performances of different networks can be compared. 

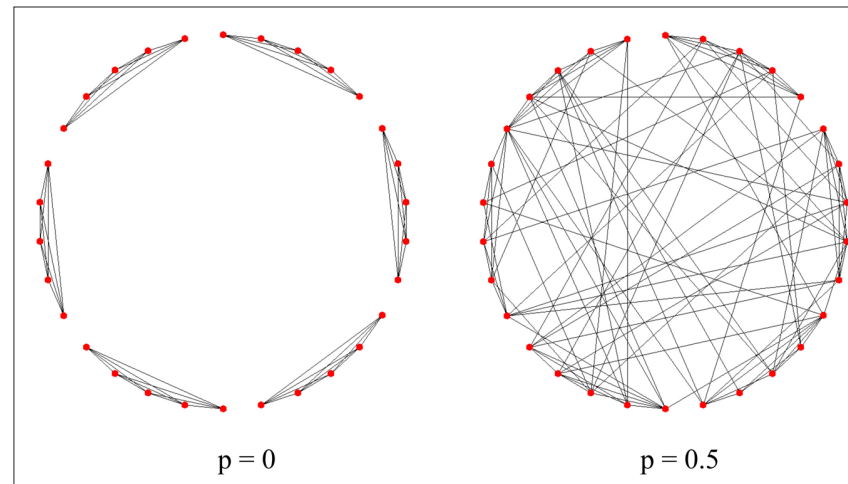

FIGURE 2 |The construction of a Fully Connected Modular network. Each network has 30 units and four afferent connections to each unit. The network is initialized as six discrete modules with fully connected internal networks (left, with rewiring rate $p=0$ ). To connect these modules, internal connections are then rewired randomly across the whole network (right, with rewiring rate $p=0.5$ ). Note that the regularity of the network is maintained during the rewiring (each node always has four afferent connections).

\section{The neuron model, learning, and dynamics}

The best connection strategy for an associative memory network may be dependent on details of the neuron model. For instance, the wiring length of a connection may have no significant impact on simple threshold neuron models, but is important in a spiking model simulating geometry-dependent time delays. To reveal the intrinsic principles that may govern network construction, this paper investigates connection strategies in various associative memory networks with either the traditional threshold unit models or the more biologically realistic, leaky integrate-and-fire spiking neuron models.

Each model needs to be trained before any measure of performance can be obtained. Canonical associative memory models with threshold units, for example the Hopfield net, commonly use a one-shot Hebbian learning rule. This, however, does not perform well if the networks are sparse and non-symmetric (Davey et al., 2006). In this paper, we adapt a more useful approach using standard perceptron learning.

A set of random, bipolar, or binary vectors is presented as training patterns, where the probability of any bit on the pattern being on $(+1)$, referred to as the bias of the pattern, is 0.5 . The patterns can be learned by the following learning rule:

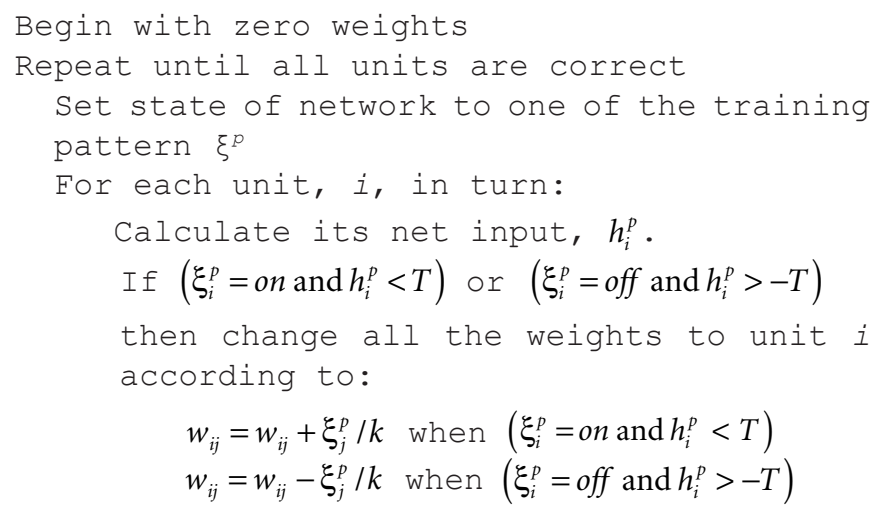

The value $\xi_{i}^{p}=$ on denotes the $i$ th bit of pattern $p$ being +1 , and the value $\xi_{i}^{p}=$ off denotes the value -1 or 0 according to the type of network. Network performance improves till saturation when the learning threshold $T$ increases, irrespective of the value of $k$. However, increasing $T$ also means a longer training time, and the improvement is insignificant for very high values of $T$. Here we set $T=10$, based on results from our previous study (Davey and Adams, 2004).

For threshold unit models we use the standard bipolar $+1 /-1$ representation. For the spiking networks we use 1/0 binary patterns, as they can be easily mapped onto the presence or absence of spikes.

Threshold unit models and integrate-and-fire spiking neuron models require different update dynamics. For threshold unit models, we use the standard asynchronous dynamics: units output +1 if their net input is positive and -1 if negative. As the connectivity is not symmetrical there is no guarantee that the network will converge to a fixed point, but, in practice these networks normally exhibit straightforward dynamics (Davey and Adams, 2004) and converge within hundreds of epochs (one epoch is a full update of every unit in the network in a fixed order). We arbitrarily set a hard limit of 5000 epochs, at which we take the network state as final even if it may not be at a fixed point. Networks with spiking neurons, on the other hand, were numerically integrated, and their state was evaluated after $500 \mathrm{~ms}$ (see below).

Due to the intrinsic model complexity, associative memory models with spiking units require careful tuning so that the performance is comparable to threshold unit models. We use a leaky integrate-and-fire neuron model which includes synaptic integration, conduction delays, and external current charges. The membrane potential $V$ of each neuron in the network is set to a resting membrane potential of $0 \mathrm{mV}$ if no stimulation is presented. The neuron can be stimulated and change its potential by either receiving spikes from other connected neurons, or by receiving externally applied current. If the membrane potential of a neuron reaches a fixed firing threshold, $T_{\text {firing }}$, which is set to $20 \mathrm{mV}$, the neuron emits a spike and the potential is reset to the resting state $(0 \mathrm{mV})$ for a certain period (the refractory period, set to $3 \mathrm{~ms}$ ). During this period the neuron cannot fire another spike even if it receives very strong stimulation.

A spike that arrives at a synapse triggers a current; the density of this current (in $A / F), I_{i j}(t)$, is given by

$I_{i j}(t)=\left(\frac{t-\left(t_{\text {spike }}+\text { delay }_{i j}\right)}{\tau_{s}}\right) \exp \left(1-\frac{t-\left(t_{\text {spike }}+\text { delay }_{i j}\right)}{\tau_{s}}\right)$

where $i$ refers to the postsynaptic neuron and $j$ to the presynaptic neuron. $\tau_{s}=2 \mathrm{~ms}$ is the synaptic time constant, $t_{\text {spike }}$ is the time when the spike is emitted by neuron $j$, and $t_{\text {spike }}+$ delay $_{i j}$ defines the time when the spike arrives at neuron $j$. Two delay modes were implemented in our study. The fixed-delay mode gives each connection a fixed $1 \mathrm{~ms}$ delay. In the second mode, the delay of spikes in a connection is defined by

$\operatorname{delay}_{i j}=\sqrt[3]{d_{i j}} \mathrm{~ms}$

where $d_{i j}$ is the connection distance which is defined as the minimum number of steps between neuron $i$ and $j$ along the ring, since the distance between neighboring neurons is 1, as described in Section "The Network Model for Associative Memory." The formula is a rough mapping from a one dimensional structure to a realistic 3-D system. 
The change of membrane potential is defined by

$\frac{d V}{d t}=-\left(\frac{V}{\tau_{m}}\right)+\sum_{i \neq j \in G} C_{i j} I_{i j} W_{i j}+I_{\text {external }}$

In this equation, $-\left(V / \tau_{m}\right)$ is the leak current density, while $\tau_{m}=50 \mathrm{~ms}$ is the membrane time constant. $I_{\text {external }}$ is the external current density which will be discussed later. The internal current is summed by $\sum_{i \neq j \in G} C_{i j} I_{i j} W_{i j}$ where $C_{i j}=1$ indicates the presence of a connection from $j$ to $i$, and $C_{i j}=0$ otherwise. $W_{i j}$ is the weight of the connection from neuron $j$ to $i$ trained by the above learning rule.

In networks trained with a learning threshold $T=10$, a single spike from an excitatory (positive-weight) connection generates a postsynaptic potential (EPSP) of about $3 \sim 4 \mathrm{mV}$ on average, and inhibitory (negative-weight) connections generate similar voltage deflections of negative sign (IPSPs). Since the proportion of excitatory and inhibitory connections is balanced (the network is trained with unbiased patterns), it requires the excess of six to seven spikes at excitatory connections to trigger the firing of a unit. Scaling down the connection weights to reduce the EPSPs also reduces the IPSPs proportionally, resulting in little or no improvement on the network's associative memory performance. The whole network is silenced, however, when all weights are scaled down to less than $20 \%$ of their original trained values, due to the current leak in the neuron model.

External currents are injected into the network in order to trigger the first spikes in the simulation. Each current injection transforms a static binary pattern to a set of current densities. Given an input pattern, unit $i$ receives an external current if it is on in that pattern, otherwise the unit receives no external current. An external current has a density of $3 A / F$ and is continually applied to the unit for the first $50 \mathrm{~ms}$ of simulation. This mechanism guarantees that the first spiking pattern triggered in the network reflects the structure of the input pattern. After the first spikes (about 7-8 ms from the start of a simulation), both internal currents caused by spikes, and the external currents, affect the network dynamics. Spike activity continues after the removal of external currents, as the internal currents caused by spike trains become the driving force. The network is then allowed to run for $500 \mathrm{~ms}$, after which time the network activity has stabilized, before its spiking pattern is evaluated.

\section{MEASURES OF MEMORY PERFORMANCE}

The associative memory performance of the threshold unit network is measured by its effective capacity (EC; Calcraft, 2005). EC is a measure of the maximum number of patterns that can be stored in the network with reasonable pattern correction still taking place. In other words, it is a capacity measure that takes into account the dynamic ability of the network to perform pattern correction. We take a fairly arbitrary definition of reasonable as the ability to correct the addition of $60 \%$ noise (redrawing $60 \%$ of the pattern bits) to within a similarity of $95 \%$ with the original fundamental memory, that is, $95 \%$ of the bits are identical. Varying these $2 \%$ figures gives differing values for EC but the values with these settings are robust for comparison purposes. For large fully connected networks the EC value is about 0.1 of the conventional capacity of the network, but for networks with sparse, structured connectivity EC is dependent upon the actual connectivity pattern.
The EC of a particular network is determined as follows:

Initialize the number of patterns, $P$, to 0

Repeat

\section{Increment $P$}

Create a training set of $P$ random patterns

Train the network

For each pattern in the training set

Degrade the pattern randomly by adding $60 \%$ of noise

With this noisy pattern as start state, allow the network to converge Calculate the similarity of the final network state with the original pattern

EndFor

Calculate the mean pattern similarity over all final states until the mean pattern similarity is less than 95\% The Effective Capacity is then P-1.

The EC of the network is therefore the highest pattern loading for which a $60 \%$ corrupted pattern has, after convergence, a mean similarity of $95 \%$, or greater with its original value.

The EC measure needs modification to suit the spiking model. We adopt the concept of memory retrieval from Anishchenko and Treves (2006). For $p$ training patterns, the memory retrieval, $M$, is defined by

$M=\frac{1}{p} \sum_{\mu=1}^{p}\left[\frac{O^{\mu}(t)-O_{\text {chance }}^{\mu}(t)}{1-O_{\text {chance }}^{\mu}(t)}\right]$

$O^{\mu}(t)$ is the overlap of the network activity and pattern $\mu$ at time $t$, more particularly the cosine of the angle between both vectors,

$O^{\mu}(t)=\frac{\sum_{i} \xi_{i}^{\mu} r_{i}(t)}{\sqrt{\sum_{i}\left[\xi_{i}^{\mu}\right]^{2} \times \sum_{i}\left[r_{i}(t)\right]^{2}}}$

where $r_{i}(t)$ is the number of spikes emitted by unit $i$ during a time window $\left[t-t_{w}, t\right]$ (we use a window of $10 \mathrm{~ms}$ ). $O_{\text {chance }}^{\mu}(t)$ is the chance level of overlap with other patterns when the external currents are injected to the network based on pattern $\mu$

$O_{\text {chance }}^{\mu}(t)=\frac{1}{p-1} \sum_{\mu^{\prime} \neq \mu} O^{\mu^{\prime}}(t)$

The memory retrieval measure was designed for sparse patterns where the chance overlap is important. In our case the patterns are unbiased so the chance overlap is close to 0 . The memory retrieval $M$ ranges between 1 and -1 , in which a high value indicates better performance. The $95 \%$ pattern similarity in the threshold unit model corresponds to a 0.9 memory retrieval value $M$ in the spiking neuron model, thus we use these two values as the EC criteria in this investigation.

\section{CONNECTIVITY MEASURES}

In recent years connectivity measures from Graph Theory have been used in the investigation of both biological and artificial neural networks. We use two of these measures to quantify our connectivity strategies.

A common measure of network connectivity is Mean Path Length. The Mean Path Length of a network $G, L(G)$, is defined as:

$L(G)=\frac{1}{N(N-1)} \sum_{i \neq j \in G} p_{i j}$ 
where $p_{i j}$ is the length of the shortest connecting path from unit $j$ to unit $i$. It is important to note here that the length of a path in the graph $G$ is the number of edges that make up the path - it has no relation to the physical distance between the nodes, $d_{i j}$.

The Mean Path Length was originally used to define the "smallworld" phenomenon found in social science (Milgram, 1967). This refers to the idea that, if a person is one step away from each person they know and two steps away from each person who is known by one of the people they know, then everyone is an average of six steps away from each person in a region like North America. Hence everyone is fairly closely related to everyone else giving a "small-world."

A fully connected network has the shortest and unique Mean Path Length of 1, whilst in sparse networks the measure varies for different types of connectivity (see Tables in Appendix). A locally connected network has a high Mean Path Length, since each unit is only connected to its nearest neighbors and it is difficult to reach distal units. On the other hand, completely random networks usually have short Mean Path Lengths. Intermediate cases, for example the Watts-Strogatz small-world network, have Mean Path Lengths similar to completely random networks, but significantly lower than the ones of lattices.

Although commonly used as a connectivity measure of neural networks, our investigation reveals that the Mean Path Length is insensitive to connectivity changes if the network is far from local. Another measure, named the Clustering Coefficient, is found to be more sensitive to connectivity changes, and exhibits a good correlation with the associative memory performance.

The definition of Clustering Coefficient is as follows. First we define neighbors of a node in a graph as its directly connected nodes.

Then we define $G_{i}$ as the subgraph of the neighbors of node $i$ (excluding $i$ itself). $C_{i}$, the local Clustering Coefficient of node $i$, is defined as:

$C_{i}=\frac{\# \text { of edges in } G_{i}}{\text { maximum possible \# of edges in } G_{i}}$

which denotes the fraction of all possible edges of $G_{i}$ which exist. The Clustering Coefficient of the graph $G, C(G)$, is then defined as the average of $c_{i}$, over all nodes of $G$ :

$C(G)=\frac{1}{N} \sum_{i \in G} C_{i}$

The Clustering Coefficient of a fully connected network is 1, since each node is connected to all others directly. Locally connected sparse networks have high Clustering Coefficients whilst in a completely random networks it is usually low. Interestingly, the so-called small-world networks usually have high Clustering Coefficients, similar to local networks, but short Mean Path Lengths, like completely random networks. Such types of connectivity (short Mean Path Length, high Clustering Coefficient) are also observed in natural networks, for instance, the mammalian cortex.

\section{RESULTS}

All networks in our study have 5000 units. The first set of results are for the non-spiking model with 250 incoming connections per unit, that is, $N=5000$ and $k=250$. The theoretical maximum capacity of such a network with threshold units is known to be $2 k=500$ for unbiased random patterns, although in practice the capacity reduces due to pattern correlations. As mentioned in Section "The Neuron Model, Learning, and Dynamics," we set the learning threshold at $T=10$ and the maximum number of updating epochs at 5000. For each network connectivity, results are averages over 10 runs. Errors bars are too small to be visible and therefore are omitted from the figures. The characteristics of different networks and their EC performances are tabulated in Section "Appendix." The first column of each table contains the values of the independent variable that was varied to construct different networks with the same connection strategy.

\section{PATH LENGTH AS A PREDICTOR OF PERFORMANCE}

As pattern correction is a global computation intuitively one might expect networks with shorter mean path lengths to perform better than those with longer mean path lengths. Our results, shown in Figure $3 \mathrm{~A}$ to some extent confirm this. The two networks with only local connections (to the right of the graph) and thereby long path lengths, do perform poorly. However path length does not discriminate amongst the majority of network architectures. This is mainly due to the fact that mean path length saturates very quickly once a few long-range connections, which act as global shortcuts, are introduced to a local network. The Tables in Section "Appendix" show that almost all the networks examined here have very similar mean path lengths of around two steps, in spite of the great variation of the independent variables.

\section{CLUSTERING COEFFICIENT AS A PREDICTOR OF PERFORMANCE}

Once again intuition suggests that performance could be related to clustering. In a highly clustered network global computation could be difficult: with information staying within clustered subnetworks and not passing through the whole network. This is confirmed by our results in Figure 3B. The networks plotted on the left side of the graph, which are highly clustered, show poor performance. But perhaps what is most noticeable about these results is the obvious linear correlation between the clustering coefficient and performance. So the clustering of a network, regardless of the details of its architecture (small-world, modular, Gaussian) completely predicts its ability to perform as an effective associative memory. A purely static property of the connection matrix appears to determine the ability of a sparsely connected recurrent neural network to perform pattern completion. We will return to why this might be the case in the discussion.

\section{THE INTEGRATE-AND-FIRE NETWORK MODEL}

We now examine how well our results generalize to the more complicated integrate-and-fire model. Because the EC evaluation of this network takes much longer to compute than for the threshold unit model, we reduced the number of incoming connections per unit, $k$, to 100. For the same reason, only the Watts-Strogatz SmallWorld network, the Gaussian network, and the Fully Connected Modular network were simulated using integrate-and-fire models. It is interesting to study whether the non-spiking network provides a qualitative and/or quantitative prediction of the much more complex spiking model. Figure 4 compares the performance of three different types of dynamics: non-spiking, spiking with fixed-delay, 


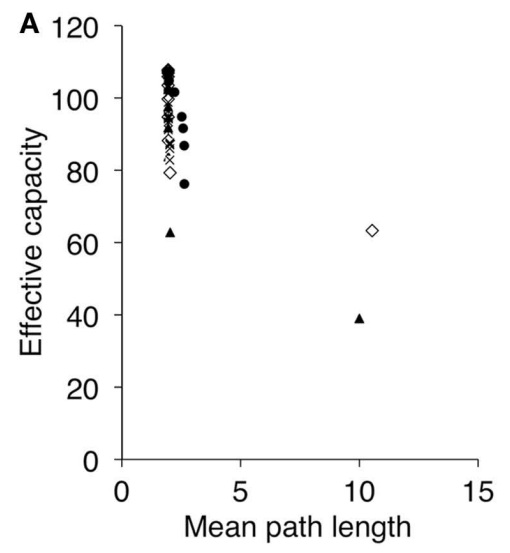

FIGURE 3 | Connectivity measures and memory performance of nonspiking network models. (A) Effective Capacity against Mean Path Length for Watts-Strogatz small-world (with varying amounts of rewiring), GaussianDistributed and Fully Connected Modular connectivity strategies. The two local networks have a significantly higher Mean Path Length of about 10, whilst Mean

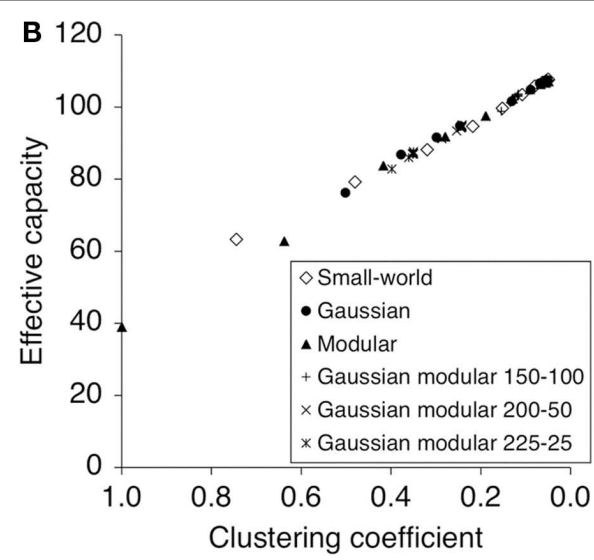

Path Lengths for all other networks are low and do not vary greatly. (B) Effective Capacity against Clustering Coefficient for a variety of networks. Number labels of different series indicate the $k_{\text {internal }}-k_{\text {external }}$ configuration of that network series. The correlation is highly similar and linear for different types of connectivity.

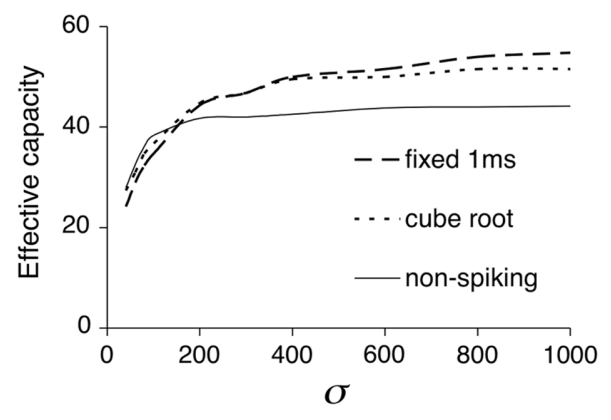

FIGURE 4 | A comparison of the non-spiking network and two versions of the spiking network. All networks have Gaussian connectivity with varying $\sigma$. The differences between the three types of the network for large $\sigma$ are significant. Results for other networks can be found in Section "Appendix."

and spiking with distance-dependent delays. The comparison is illustrated for Gaussian connectivity patterns. Similar comparisons can be made for other connection strategies from the data given in Section "Appendix." The connectivity of the networks furthest to the left has a very tight Gaussian distribution $(\sigma=0.4)$. Interestingly all three networks give a similar EC value of about 25. In all three networks performance then improves as some distal connections are introduced, until the non-spiking network attains its best performance of $\mathrm{EC}=42$. However the spiking networks show further improvements reaching EC values of over 50. But perhaps the most striking feature of this result is that the simple threshold model provides a good approximation of the much more complex spiking neural network.

Figure 5 presents the results of EC against Clustering Coefficient for spiking models with fixed time delay (1 ms) and cube-root delay. The linear correlation between EC and Clustering Coefficient is not absolutely maintained in the spiking models, however the results still show that the EC increases as the Clustering Coefficient decreases. In the fixed-delay models, the performance of all three
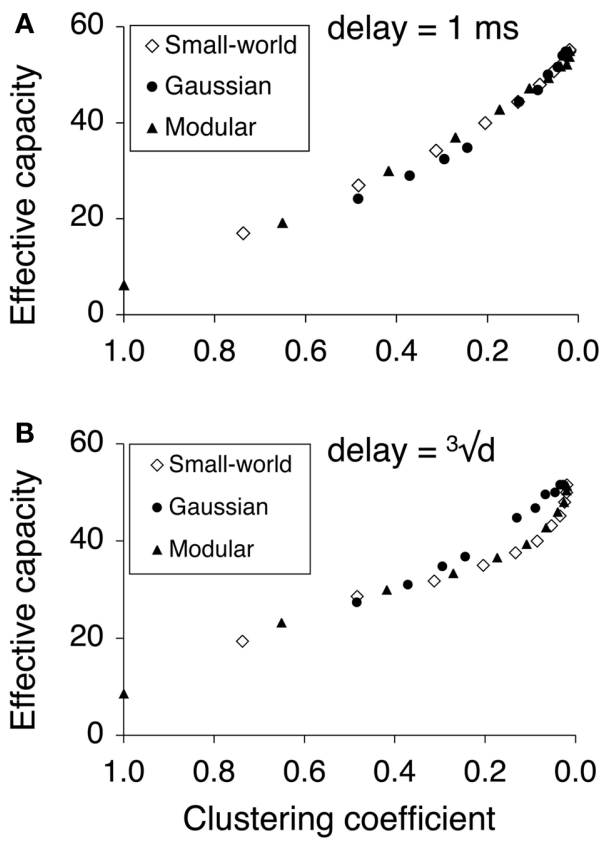

FIGURE 5 | Correlation between Effective Capacity and Clustering Coefficient for spiking network models. For detailed results see Section "Appendix." (A) Fixed 1 ms delay spiking associative memory models with different connectivity. (B) Cube-root distance delay.

patterns of connectivity is very similar, whilst in the cube-root delay models, the performance of the Gaussian connectivity varies more linearly than that of the other two connectivities. This is because the other two types of connectivity have more distal connections due to their uniform rewiring, consequently increasing the distance-dependent delay of the networks. Overall the Clustering Coefficient can still be used as a reasonable predictor of associative memory performance. 


\section{CONNECTION STRATEGIES FOR OPTIMIZING WIRING COST AND PERFORMANCE}

In nature, the construction of associative memory networks is restricted by resource, thus a connection strategy that optimizes both wiring cost and performance would be preferable. We define the wiring cost of two connected nodes in the network simply as the distance between them, and average it over all connected nodes in the network. Note that this is a quite different measure to mean path length, which measures steps along the connection graph. This measure is also different from the connection delay used in the spiking network models, where the cube-root of $d$ was taken to avoid unrealistically long delays. For simplicity this section focuses on the performance of the threshold unit models.

Figure 6 plots the EC against the Mean Wiring Cost for different connectivity patterns. The Gaussian-distributed network has the most efficient connectivity, that is, for a given mean wiring cost, it has the highest EC. The fully connected Modular network is the least efficient one, whilst the Watts-Strogatz small-world network, and Gaussian-uniform Modular network lay between them. Effective Gaussian networks can be constructed with very low wiring cost because the width of the distribution can be quite small. In fact a width of just $2 k$ (here 500 ) gives good performance at low cost. We also found that the distribution does not need to increase in width as the network gets bigger (Calcraft et al., 2007).

\section{DISCUSSION}

Our study of memory performance in networks with varying connection strategies extends previous work on the effect of connectivity on network dynamics (Stewart, 2004; Percha et al., 2005), wiring economy and development (Chklovskii et al., 2004), and disease (Belmonte et al., 2004; Lynall et al., 2010; Stam, 2010).

\section{MAIN FINDINGS AND LIMITATIONS}

The main finding of the present study is that for networks with a fixed number $k$ of connections, and hence a theoretical maximum storage capacity of $2 k$ random patterns, the degree of clustering can be regarded as a negative predictor of memory performance. This

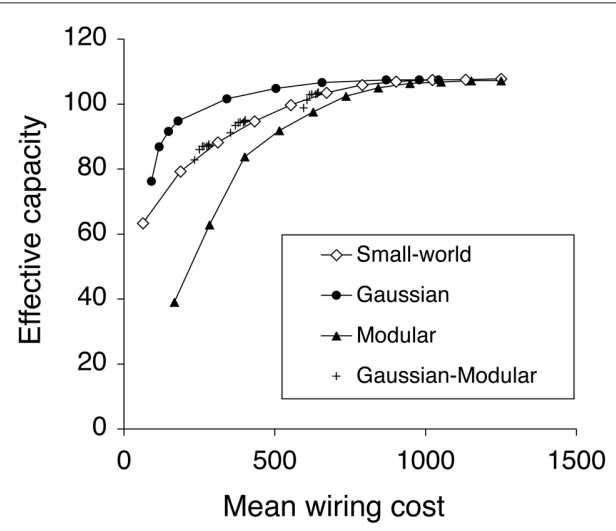

FIGURE 6 | Effective Capacity against Mean Wiring Cost for different connectivity strategies. The best networks occupy the top left of the graph. The Gaussian-Distributed network is the most efficient connectivity among those investigated connectivity, whilst the Fully Connected Modular network is the least efficient one. prediction is independent of the connection rule used. We further demonstrated that when the cost of wiring is taken into account, by penalizing long-range connections, networks with local Gaussian-distributed connections perform best, and even outperform small-world networks. Finally, these findings also hold when memory is stored and retrieved in networks of spiking neurons with connection delays.

These results were obtained with random uncorrelated patterns, hence without taking into account the statistics of a natural environment. The patterns were stationary (no sequences) and so was largely the dynamics of the network (no oscillations or temporal patterns). Our study was conducted on a ring of neurons, but the results can be extended to $2-\mathrm{D}$ networks (Calcraft et al., 2008).

In the network of spiking neurons, the resulting feedback excitation and inhibition forced the neurons into persistent UP or DOWN states. The network lacked, however, dynamical behavior like gain control, synchronization, or rhythm generation, for which separate populations of excitatory and inhibitory neurons would be required (Sommer and Wennekers, 2001; de Almeida et al., 2007). In addition, the spiking network used the same synaptic weights as obtained with the perceptron-rule for bipolar neurons, and was only tested for memory retrieval. Learning in such networks would require a more advanced algorithm like spike-timing-dependent plasticity.

\section{CLUSTERING AS A NEGATIVE PREDICTOR OF MEMORY PERFORMANCE}

Clustering (the occurrence of connections between the targets of the same neuron) may make some connections functionally redundant or induce loops. Cycling through loops may hamper convergence to an attractor. In addition, Zhang and Chen (2008) formally demonstrated that loops also induce correlations between states, or equivalently, they induce noise in the form of an interpattern crosstalk term to a neuron's input, and hence affect pattern separation. Note that this does not directly imply that inducing clustering by adding local connections would worsen memory performance, since the added connections would increment $k$ and hence the maximum capacity as well.

\section{MEMORY STORAGE WITH LOCAL CONNECTIVITY}

An unexpected finding was that for a given wiring cost, Gaussianconnected networks performed better than small-world networks. Although very-long-range connections speed up the convergence to an attractor during memory retrieval (Calcraft et al., 2007), they do not enhance the steady-state performance of the network. Moreover, the width (SD) of the Gaussian connection kernel can be small (Figure 4), and does not scale with network size.

\section{RELATION TO BRAIN CONNECTIVITY}

The connectivity of the brain, and more particularly of neocortex, is sparse, but certainly not random (see Laughlin and Sejnowski, 2003). Counting the close appositions between axonal and dendritic trees of reconstructed pyramidal cells assembled in a computer network, Hellwig (2000) estimated that connection probability decreased with inter-neuron distance as a Gaussian function of about $500 \mu \mathrm{m}$ width (the order of magnitude of a cortical column; see Figure 7). Recent experiments using distant release of caged glutamate by photo-stimulation extend this range, indicating a still considerable excitation from distances up to $2 \mathrm{~mm}$ (Schnepel et al., 2010). 


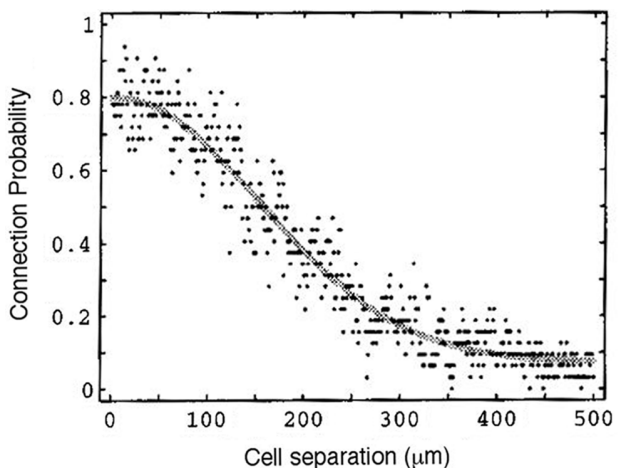

FIGURE 7 | Estimated connection probability between two layer-3 pyramidal neurons of rat visual cortex. Copied from Hellwig (2000) with permission of Springer-Verlag. The best-fitting Gaussian has a SD of $296.7 \mu \mathrm{m}$.

Within a column, Song et al. (2005) found an overrepresentation of bi-directional connections and local three-neuron clusters in rat visual cortex. Whereas such clustering may be a sign

\section{REFERENCES}

Anishchenko, A., and Treves, A. (2006). Autoassociative memory retrieval and spontaneous activity bumps in smallworld networks of integrate-and-fire neurons. J. Physiol. (Paris) 100,225-236.

Belmonte, M. K., Allen, G., BeckelMitchener,A., Boulanger,L.M., Carper, R. A., and Webb, S. J. (2004). Autism and abnormal development of brain connectivity. J. Neurosci. 24,9228-9231.

Brucoli, M., Carnimeo, L., and Grassi, G. (1996). "Implementation of cellular neural networks for heteroassociative and autoassociative memories," Proceedings of the Fourth IEEE International Workshop on Cellular Neural Networks and their Applications 1996, Seville, 63-68.

Calcraft, L. (2005). "Measuring the performance of associative memories," in Computer Science Technical Report 420, University of Hertfordshire, Hatfield, Herts.

Calcraft,L.,Adams, R., and Davey,N. (2006). Locally-connected and small-world associative memories in large networks. Neural Inf. Process. Lett. Rev. 10, 19-26.

Calcraft,L.,Adams, R., and Davey,N. (2007). Efficient architectures for sparselyconnected high capacity associative memory models. Conn. Sci. 19, 163-175.

Calcraft, L., Adams, R., and Davey, N. (2008). Efficient connection strategies in $1 \mathrm{D}$ and $2 \mathrm{D}$ associative memory models with and without displaced connectivity. Biosystems 94, 87-94.

Chen, W., Maex, R., Adams, R., Steuber, V., Calcraft, L., and Davey, N. (2009). "Connection strategies in associative memory models with spiking and non-spiking neurons," in Adaptive and Natural Computing Algorithms, eds M. Kolehmainen, P. Toivanen, and B.
Beliczynski (Berlin: Springer Verlag), 42-51. [Lecture Notes in Computer Science 5495].

Chklovskii, D. B., Mel, B. W., and Svoboda, K. (2004). Cortical rewiring and information storage. Nature 431, 782-788.

Davey, N., and Adams, R. (2004). High capacity associative memories and connection constraints. Conn. Sci. 16,47-66.

Davey,N., Calcraft,L., and Adams, R. (2006). High capacity, small world associative memory models. Conn. Sci. 18, 247-264.

de Almeida, L., Idiart, M., and Lisman, J. E. (2007). Memory retrieval time and memory capacity of the CA3 network: role of gamma frequency oscillations. Learn. Mem. 14, 795-806.

Diederich, S., and Opper, M. (1987). Learning of correlated patterns in spin-glass networks by local learning rules. Phys. Rev. Lett. 58, 949-952.

Gardner, E. (1988). The space of interactions in neural network models. J. Phys. A 21, 257-270.

Gerhard,F.,Pipa,G., and Gerstner,W.(2010). Estimating small-world topology of neural networks from multi-electrode recordings. Front. Comput. Neurosci. doi: 10.3389/conf.fncom.2010.51.00088.

Hellwig, B. (2000).A quantitative analysis of the local connectivity between pyramidal neurons in layers $2 / 3$ of the rat visual cortex. Biol. Cybern. 82, 111-121.

Hopfield, J.J. (1982). Neural networks and physical systems with emergent collective computational abilities. Proc. Natl. Acad. Sci. U.S.A. 79, 2554-2558.

Humphries, M. D., and Gurney, K. (2008). Network "small-world-ness": a quantitative method for determining canonical network equivalence. $P L O S$ ONE 3, e0002051. doi: 10.1371/journal.pone. 0002051

of a genuine small-world connectivity, the observed clustering of connections may also have a function in the dynamics of the network, or be a consequence of the multidimensional representation of the visual field onto neocortex, mapping not only stimulus position but also higher-order stimulus attributes like orientation. Nevertheless, neocortex is assumed to have a small, but significant, small-worldness (Gerhard et al., 2010), as based on a metric using the ratio of clustering over relative path length (Humphries and Gurney, 2008).

\section{CONCLUSION}

We studied the capacity of memory storage and retrieval in networks of bipolar and spiking neurons, and compared different connection strategies and connection metrics. The single metric best predicting memory performance, for all strategies, was the clustering coefficient, with performance being highest when clustering was low. In large networks, the best connection strategy was a local Gaussian probability function of distance, both in terms of avoiding clustering and of minimizing the cost of wiring.

Laughlin, S. B., and Sejnowski, T. J. (2003). Communication in neuronal networks. Science 301, 1870-1874.

Lynall, M. E., Bassett, D. S., Kerwin, R. McKenna, P. J., Kitzbichler, M., Muller, U., and Bullmore, E. (2010). Functional connectivity and brain networks in schizophrenia. J. Neurosci. 30, 9477-9487.

Marr, D. (1969). A theory of cerebellar cortex. J. Physiol. 202, 437-470.

Milgram, S. (1967). The Small World Problem. Psychol. Today 1, 60-67.

Mountcastle, V. B. (1997). The columnar organization of the neocortex. Brain 120, 701-722.

Percha, B., Dzakpasu, R., Zochowski, M., and Parent, J. (2005). Transition from local to global phase synchrony in small world neural network and its possible implications for epilepsy. Phys. Rev. E Stat. Nonlin. Soft Matter Phys. 72, 031909.

Schnepel, P., Nawrot, M., Aertsen, A., and Boucsein,C. (2010). Number, reliability and precision of long-distance projections onto neocortical layer 5 pyramidal neurons. Front. Comput. Neurosci. doi: 10.3389/conf.fncom.2010.51.00110 [Epub ahead of print].

Sommer, F. T., and Wennekers, T. (2001). Associative memory in networks of spiking neurons. Neural Netw. 14, 825-834.

Song, S., Sjöström, P. J., Reigl, M., Nelson, S. and Chklovskii, D.B.(2005). Highly nonrandom features of synaptic connectivity in local cortical circuits. PLoS Biol. 3, e68. doi: 10.1371/journal.pbio.0030068 [Erratum in: PLoS Biol. 3, e350].

Stam, C. J. (2010). Characterization of anatomical and functional connectivity in the brain: a complex networks perspective. Int. J. Psychophysiol. 77, 186-194.
Steuber, V., Mittmann, W., Hoebeek, F. E., Silver, R. A., De Zeeuw, C. I., Hausser, M., and De Schutter, E. (2007). Cerebellar LTD and pattern recognition by Purkinje cells. Neuron 54, 121-136.

Stewart, I. (2004). Networking opportunity. Nature 427, 601-604.

Tyrrell, L. R. T., and Willshaw, D. J. (1992). Cerebellar cortex: its simulation and the relevance of Marr's theory. Philos. Trans. R. Soc. Lond. B Biol. Sci. 336, 239-257.

Watts, D. J., and Strogatz, S. H. (1998). Collective dynamics of "small-world" networks. Nature 393, 440-442.

Zhang, P., and Chen, Y. (2008). Topology and dynamics of attractor neural networks: the role of loopiness. Physica A 387, 4411-4416.

Conflict of Interest Statement: The authors declare that the research was conducted in the absence of any commercial or financial relationships that could be construed as a potential conflict of interest.

Received: 14 October 2010; accepted: 17 March 2011; published online: 30 March 2011.

Citation: Chen W, Maex R, Adams R, Steuber V, Calcraft L and Davey N (2011) Clustering predicts memory performance in networks of spiking and non-spiking neurons. Front. Comput. Neurosci. 5:14. doi: 10.3389/fncom.2011.00014

Copyright (c) 2011 Chen, Maex, Adams, Steuber, Calcraft and Davey. This is an open-access article subject to a nonexclusive license between the authors and Frontiers Media SA, which permits use, distribution and reproduction in other forums, provided the original authors and source are credited and other Frontiers conditions are complied with. 


\section{APPENDIX}

\section{NON-SPIKING NETWORK RESULTS}

Network size: 5000 units, 250 connections per unit.

W-S small-world network.

\begin{tabular}{lrrrr}
\hline Rewiring & MPL & CC & WC & EC \\
\hline 0 & 10.540 & 0.745 & 63 & 63.3 \\
0.1 & 2.030 & 0.481 & 187 & 79.3 \\
0.2 & 1.959 & 0.320 & 311 & 88.2 \\
0.3 & 1.951 & 0.218 & 432 & 94.7 \\
0.4 & 1.950 & 0.152 & 553 & 99.7 \\
0.5 & 1.950 & 0.108 & 672 & 103.5 \\
0.6 & 1.950 & 0.080 & 790 & 105.9 \\
0.7 & 1.950 & 0.063 & 902 & 106.9 \\
0.8 & 1.950 & 0.054 & 1022 & 107.4 \\
0.9 & 1.950 & 0.050 & 1132 & 107.5 \\
1 & 1.950 & 0.050 & 1250 & 107.8 \\
\hline
\end{tabular}

Gaussian network.

\begin{tabular}{|c|c|c|c|c|}
\hline$\sigma$ & MPL & CC & WC & EC \\
\hline 0.4 & 2.632 & 0.501 & 91 & 76.2 \\
\hline 0.6 & 2.626 & 0.378 & 117 & 86.8 \\
\hline 0.8 & 2.580 & 0.299 & 147 & 91.6 \\
\hline 1 & 2.522 & 0.247 & 179 & 94.8 \\
\hline 2 & 2.218 & 0.131 & 341 & 101.6 \\
\hline 3 & 1.989 & 0.089 & 504 & 104.8 \\
\hline 4 & 1.952 & 0.069 & 656 & 106.6 \\
\hline 6 & 1.950 & 0.054 & 869 & 107.4 \\
\hline 8 & 1.9502 & 0.052 & 979 & 107.4 \\
\hline 10 & 1.9502 & 0.051 & 1043 & 107.4 \\
\hline \multicolumn{5}{|c|}{ Fully connected modular network. } \\
\hline Rewiring & MPL & CC & WC & EC \\
\hline 0 & undefined & 1.000 & 167 & 39 \\
\hline 0.1 & 2.035 & 0.638 & 284 & 62.8 \\
\hline 0.2 & 1.959 & 0.418 & 400 & 83.8 \\
\hline 0.3 & 1.951 & 0.279 & 515 & 91.8 \\
\hline 0.4 & 1.950 & 0.189 & 627 & 97.6 \\
\hline 0.5 & 1.950 & 0.129 & 736 & 102.4 \\
\hline 0.6 & 1.950 & 0.091 & 843 & 105 \\
\hline 0.7 & 1.950 & 0.067 & 948 & 106.4 \\
\hline 0.8 & 1.950 & 0.055 & 1051 & 106.8 \\
\hline 0.9 & 1.950 & 0.050 & 1151 & 107.2 \\
\hline 1 & 1.950 & 0.050 & 1250 & 107.2 \\
\hline
\end{tabular}

Gaussian modular network.

\begin{tabular}{|c|c|c|c|c|}
\hline$\sigma$ & MPL & CC & WC & EC \\
\hline \multicolumn{5}{|c|}{$\begin{array}{l}\text { INTRA-MODULAR CONNECTIONS = 150, } \\
\text { INTERMODULAR CONNECTIONS = } 100 .\end{array}$} \\
\hline 0.4 & 1.950 & 0.154 & 595 & 98.8 \\
\hline 0.6 & 1.950 & 0.135 & 607 & 101.2 \\
\hline 0.8 & 1.950 & 0.124 & 616 & 102.8 \\
\hline 1 & 1.950 & 0.119 & 623 & 103 \\
\hline 2 & 1.950 & 0.117 & 636 & 103 \\
\hline 4 & 1.950 & 0.117 & 641 & 103.2 \\
\hline 8 & 1.950 & 0.117 & 643 & 103.6 \\
\hline 10 & 1.950 & 0.116 & 644 & 103.6 \\
\hline
\end{tabular}

INTRA-MODULAR CONNECTIONS $=\mathbf{2 0 0 ,}$ INTERMODULAR CONNECTIONS = 50.

$\begin{array}{lllll}0.4 & 1.957 & 0.287 & 353 & 91.2 \\ 0.6 & 1.957 & 0.254 & 369 & 93.4 \\ 0.8 & 1.957 & 0.245 & 379 & 94.2 \\ 1 & 1.957 & 0.242 & 385 & 94.4 \\ 2 & 1.957 & 0.242 & 396 & 94.4 \\ 4 & 1.957 & 0.242 & 400 & 94.6 \\ 8 & 1.957 & 0.242 & 402 & 94.8 \\ 10 & 1.957 & 0.242 & 402 & 95\end{array}$

\begin{tabular}{|c|c|c|c|c|}
\hline \multicolumn{5}{|c|}{$\begin{array}{l}\text { INTRA-MODULAR CONNECTIONS = 225, } \\
\text { INTERMODULAR CONNECTIONS = } 25 .\end{array}$} \\
\hline 0.4 & 2.019 & 0.398 & 234 & 82.8 \\
\hline 0.6 & 2.020 & 0.361 & 250 & 86 \\
\hline 0.8 & 2.020 & 0.353 & 260 & 87 \\
\hline 1 & 2.020 & 0.351 & 265 & 87 \\
\hline 2 & 2.020 & 0.350 & 275 & 87 \\
\hline 4 & 2.020 & 0.350 & 280 & 87 \\
\hline 8 & 2.020 & 0.350 & 281 & 87.2 \\
\hline 10 & 2.020 & 0.350 & 282 & 87.6 \\
\hline
\end{tabular}

CC, clustering coefficient; EC, effective capacity; EC_Cuberoot, effective capacity for cube-root distance-dependent delay spiking network; EC_Fixed, effective capacity for fixed-delay spiking network; MPL, mean path length; WC, wiring cost. 


\section{SPIKING NETWORK RESULTS}

Network size: 5000 units, 100 connections per unit.

W-S small-world network.

\begin{tabular}{lrlrlll}
\hline Rewiring & MPL & CC & WC & EC & EC_Fixed & $\begin{array}{l}\text { EC_ } \\
\text { Cuberoot }\end{array}$ \\
\hline 0 & & & & & & \\
0.1 & 25.495 & 0.737 & 25 & 22 & 17 & 19.4 \\
0.2 & 2.629 & 0.484 & 138 & 28.6 & 27 & 28.6 \\
0.3 & 2.438 & 0.313 & 264 & 35.4 & 34.2 & 31.8 \\
0.4 & 2.317 & 0.205 & 390 & 39 & 40 & 35 \\
0.5 & 2.239 & 0.133 & 513 & 41.2 & 44.4 & 37.6 \\
0.6 & 2.187 & 0.085 & 638 & 42.2 & 48 & 40 \\
0.7 & 2.153 & 0.054 & 761 & 44 & 50.8 & 43.2 \\
0.8 & 2.132 & 0.035 & 884 & 44.6 & 52.4 & 45.2 \\
0.9 & 2.117 & 0.024 & 1006 & 44.4 & 54.4 & 48 \\
1 & 2.111 & 0.020 & 1129 & 44 & 54.8 & 50 \\
\hline
\end{tabular}

Gaussian network.

\begin{tabular}{lllllll}
\hline $\boldsymbol{\sigma}$ & MPL & CC & WC & EC & EC_Fixed & $\begin{array}{l}\text { EC_ } \\
\text { Cuberoot }\end{array}$ \\
\hline 0.4 & 2.889 & 0.484 & 52 & 28 & 24.2 & 27.4 \\
0.6 & 3.012 & 0.371 & 53 & 32.6 & 29 & 31 \\
0.8 & 3.102 & 0.295 & 61 & 36.2 & 32.4 & 34.8 \\
1 & 3.142 & 0.244 & 72 & 38.4 & 34.8 & 36.8 \\
2 & 3.074 & 0.130 & 131 & 41.8 & 44.4 & 44.8 \\
3 & 2.865 & 0.089 & 192 & 42 & 46.8 & 46.8 \\
4 & 2.642 & 0.067 & 254 & 42.6 & 50 & 49.6 \\
6 & 2.396 & 0.045 & 377 & 43.8 & 51.6 & 50 \\
8 & 2.274 & 0.034 & 500 & 44 & 54 & 51.6 \\
10 & 2.193 & 0.028 & 616 & 44.2 & 54.8 & 51.6 \\
\hline
\end{tabular}

Fully connected modular network.

\begin{tabular}{lcccccc}
\hline Rewiring & MPL & CC & WC & EC & EC_Fixed & $\begin{array}{l}\text { EC_ } \\
\text { Cuberoot }\end{array}$ \\
\hline 0 & & & & & & 8.6 \\
0.1 & 2.671 & 0.651 & 146 & 18.8 & 19.2 & 23.2 \\
0.2 & 2.468 & 0.418 & 271 & 31 & 30 & 30 \\
0.3 & 2.339 & 0.271 & 394 & 37.2 & 37 & 33.4 \\
0.4 & 2.256 & 0.173 & 518 & 40.1 & 42.8 & 36.6 \\
0.5 & 2.200 & 0.108 & 642 & 42 & 47.2 & 39.4 \\
0.6 & 2.164 & 0.066 & 765 & 44 & 49.4 & 42.8 \\
0.7 & 2.139 & 0.040 & 886 & 44 & 51.8 & 46 \\
0.8 & 2.124 & 0.026 & 1009 & 44.2 & 52.2 & 48 \\
0.9 & 2.116 & 0.021 & 1130 & 44 & 53.8 & 50.4 \\
1 & 2.116 & 0.020 & 1250 & 44 & 55 & 51.4 \\
\hline
\end{tabular}

CC, clustering coefficient; EC, effective capacity; EC_Cuberoot, effective capacity for cube-root distance-dependent delay spiking network; EC_Fixed, effective capacity for fixed-delay spiking network; MPL, mean path length; WC, wiring cost. 